\title{
Evaluation of the safety and efficacy of low-dose rasburicase in critically ill children with haematological malignancies
}

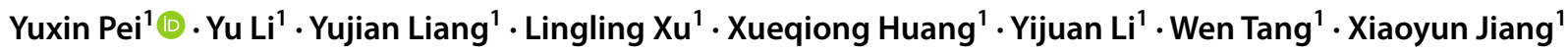

Received: 14 May 2020 / Accepted: 2 September 2020 / Published online: 24 September 2020

(c) The Author(s) 2020

\begin{abstract}
Background The recommended dose of rasburicase is quite expensive, thus limiting its use. Whether a lower dose of rasburicase would be equally effective for critically ill children, who often have more complicated situations and a higher risk of hospital death, is still unknown. Objective To explore the safety and efficacy of low-dose rasburicase in critically ill children with haematological malignancies who are at high risk of tumour lysis syndrome. Setting A single-centre retrospective cohort study. Method Children with haematological malignancies who had a history of rasburicase exposure during an intensive care unit stay were enrolled. Patients were divided into two groups according to the initial dosage of rasburicase: the standard-dose group ( $>0.1 \mathrm{mg} / \mathrm{kg} / \mathrm{day})$ and the low-dose group $(\leq 0.1 \mathrm{mg} / \mathrm{kg} / \mathrm{day})$. The adverse events and short-term prognosis of the two groups were compared. Results Thirty-seven children were selected, 22 in the standard-dose group and 15 in the low-dose group. The most common tumour type was Burkitt's lymphoma (81\%), followed by acute lymphoblastic leukaemia (11\%). All patients were at high risk of tumour lysis syndrome, and $73 \%$ of them had 3 or more tumour lysis syndrome risk factors. The uric acid levels of $90 \%$ of patients with hyperuricaemia returned to the normal range within $12 \mathrm{~h}$ (100\% in the standard-dose group and $75 \%$ in the low-dose group, $P=0.083$ ). Eighty-four percent of patients presented serious complications, including tumour lysis syndrome (73\%), acute kidney injury (59\%), renal replacement treatment (24\%), respiratory failure (24\%), disseminated intravascular coagulation (16\%) and heart failure (11\%). There was no significant difference in the incidence of serious complications between the two groups. The overall 7-day and 28-day survival rates after intensive care unit admission were $86 \%$ and $84 \%$, respectively. The average length of stay in the intensive care unit was $9.92 \pm 5.13$ days. Neither the short-term mortality nor the length of stay in the intensive care unit were significantly different between the two groups. Conclusion Low-dose rasburicase is effective and may be an acceptable choice for critically ill children with haematological malignancies.
\end{abstract}

Keywords Children $\cdot$ Haematological malignancies $\cdot$ Hyperuricaemia $\cdot$ Intensive care unit $\cdot$ Rasburicase

\section{Impacts on Practice}

- Rasburicase is recommended for children who are at high risk of tumour lysis syndrome.

- This research provides guidance on using rasburicase in children with a high tumour burden managed in the intensive care unit.

Yuxin Pei and Yu Li have contributed equally to the study.

Xiaoyun Jiang

jiangxiaoyun2015@126.com

1 The First Affiliated Hospital of Sun-yat, Sen University, Guangzhou, Guangdong Province, China
- Dosage regimes of $<0.1 \mathrm{mg}$ per kg body weight per day resulted in similar outcomes compared to dosage regimens of $>0.1 \mathrm{mg} / \mathrm{kg}$ body weight per day

- Finally, this study may provide novel study angles for dosage regimens of rasburicase, especially in resourcelimited regions.

\section{Introduction}

Tumour lysis syndrome (TLS) is an important cause of early death as well as admission to the intensive care unit (ICU) in children with newly diagnosed haematologic malignancies [1]. It is a serious metabolic syndrome that occurs due to rapid lysis of tumour cells. Lysed cells release large 
quantities of cellular contents into the blood circulation and may result in hyperkalaemia, hypocalcaemia, hyperuricaemia, hyperphosphatemia and multiple organ dysfunction [2]. Both hyperuricaemia and hyperphosphatemia can cause the deposition of crystals in the renal tubular system. This deposition may result in acute kidney injury (AKI), a serious complication associated with a poor short-term prognosis in children [3]. Children with haematologic malignancies have a high potential for cell lysis; thus, they are considered to have a high risk of developing TLS, and combined treatment regimens, including close laboratory and clinical monitoring, increased hydration and uric acid control therapy, have been proposed.

In the treatment of hyperuricaemia, urate oxidase is recommended for children at high risk of TLS because it can rapidly convert uric acid to allantoin, which is far more soluble than uric acid. Compared with those who use allopurinol, children who use rasburicase, a type of recombinant urate oxidase, are less likely to receive renal replacement therapy. The Food and Drug Administration (FDA)-approved dose of rasburicase is $0.2 \mathrm{mg} / \mathrm{kg} /$ day for up to 5 days. In clinical practice, a dosage of $0.1-0.2 \mathrm{mg} / \mathrm{kg} /$ day of rasburicase is often applied [4]. Nonetheless, this uric acid-lowering strategy is much more expensive than the traditional treatment with allopurinol [5]. For decrease costs, some researchers attempted to determine the minimum effective dose and frequency for rasburicase. A recent study showed that a fixed dose of $6 \mathrm{mg}$ rasburicase is effective for children and adolescents who have a weight exceeding $30 \mathrm{~kg}$ [6]. However, haematologic malignancies at high risk of TLS are also prevalent in young children [7, 8]. There is still limited evidence on the use of a lower dose of rasburicase, especially in critically ill children $[9,10]$. It is therefore important to explore the efficacy of low-dose rasburicase therapy in critically ill children with haematologic malignancies.

\section{Aim of the study}

The objective of this study was to explore the safety and efficacy of low-dose rasburicase in critically ill children with haematologic malignancies who were at high risk of TLS.

\section{Ethics approval}

This retrospective chart review study involving human participants was in accordance with the ethical standards of the institutional and national research committee and with the 1964 Helsinki Declaration and its later amendments or comparable ethical standards. The Medical Ethics Committee of the First Affiliated Hospital of Sun Yat-sen University approved this study [No. 2020-092].

\section{Methods}

\section{Study design and participants}

This single-centre retrospective cohort study included children with newly diagnosed haematologic malignancies who were admitted to the Paediatric Intensive Care Unit (PICU) of The First Affiliated Hospital of Sun Yat-sen University between January 2014 and January 2020. Patients $<18$ years old who had a confirmed diagnosis of haematological malignancies by histology or cytology and received at least one dose of rasburicase were eligible for inclusion. Patients who had completed induction chemotherapy or did not have rasburicase exposure during their ICU stay were excluded. Clinical information, including demographic data, tumour factors, treatment modalities, and outcomes, was collected. The definition of and assessment of risk for TLS was performed according to the British Committee for Standards in Haematology-recommended guidelines [11]. The clinical TLS risk classification proposed by Michael Darmon was used [12]. AKI was identified and staged based on the 2012 Kidney Disease Improving Global Outcome (KDIGO) definition [13]. Chemotherapy regimens were based on tumour characteristics according to the South China Children's Cancer Group (SCCCG) protocol [7, 14]. The standard treatment protocols for TLS include risk assessment, increased hydration, careful monitoring, and management of hyperuricaemia [11]. All patients were managed with increased hydration for $31 / \mathrm{m}^{2} / 24 \mathrm{~h}$ to maintain urine output $>4 \mathrm{ml} / \mathrm{kg} / \mathrm{h}$ for infants and $100 \mathrm{ml} / \mathrm{m}^{2} / \mathrm{h}$ for older children. Laboratory tests, including analyses for electrolytes, uric acid levels and renal function, were performed at least daily during the ICU stay. Two uric-acid-lowering agents were applied according to different risk stratifications: rasburicase was given prophylactically before or within the first week of initial chemotherapy for children at high risk of TLS after PICU admission, while allopurinol was given for the others. According to the daily dose of rasburicase, children were divided into two arms: the standard-dose group ( $>0.1 \mathrm{mg} / \mathrm{kg} /$ day and $\leq 0.2 \mathrm{mg} / \mathrm{kg} / \mathrm{day})$ and the low-dose group $(\leq 0.1 \mathrm{mg} / \mathrm{kg} /$ day). Follow-up information was obtained from general ward or outpatient clinic visits.

\section{Outcomes}

The 7-day and 28-day mortality rates after admission to the PICU were used to assess short-term prognosis. The efficacy of treatments with different dosages of rasburicase was evaluated by comparing the need for renal replacement therapy (RRT), the duration of hyperuricaemia, the incidence of serious complications and the length of stay in the ICU. 


\section{Statistical analysis}

Demographic and tumour features are summarized descriptively. Continuous variables are reported as the mean \pm SD or median (lower quartile: upper quartile) and analysed by two-tailed $t$ test using a Student $t$ test or Wilcoxon rank-sum test. Categorical variables are presented as actual numbers and percentages and compared by using the Chi square test, Fisher's exact test or Pearson's Chi square test as appropriate. Overall survival curves were plotted using the KaplanMeier method. $P$ values less than 0.05 were considered significant, and actual values are shown in each table. Statistical analyses were carried out with SPSS version 16.0 and GraphPad Prism version 5.03.

\section{Results}

\section{Clinical Characteristics}

From January 2014 to January 2020, 65 critically ill children with newly diagnosed haematological malignancies were admitted to our centre. Twenty-eight patients who did not have rasburicase exposure were excluded. Thirtyseven patients were eligible to be included in this study; 28 were boys, and 9 were girls. The mean age at diagnosis was $6.4 \pm 3.4$ years, with a mean disease course of $35 \pm 26$ days. Most of them had Burkitt's lymphoma (30/37, 81\%). The remaining cases were of lymphoblastic lymphoma (2/37, $5 \%)$, acute lymphoblastic leukaemia $(4 / 37,11 \%)$ and acute myeloid leukaemia $(1 / 37,2 \%)$.

The main reasons for ICU admission were dyspnoea or acute respiratory failure (41\%), abdominal compartment syndrome (27\%) and monitoring (24\%). Hyperuricaemia was present on admission in $81 \%$ of cases. The baseline lactate dehydrogenase (LDH) was $2179(1137,3282) \mathrm{U} / \mathrm{L}$, and the peak LDH often appeared on D3-D5 of pre-phase chemotherapy, with a median value of $2936(1697,6497) \mathrm{U} / \mathrm{L}$. Older children or those with higher weights preferred to take lower initial doses of rasburicase. In addition, there was no difference in baseline characteristics between the standarddose group and the low-dose group. The demographic data and tumour characteristics of the two groups are summarized in Table 1.

Based on the risk assessment for malignant disease type, all patients were at high risk of TLS, $14 \%(n=5)$ had acute lymphoblastic leukaemia or acute myeloid leukaemia with an elevated white blood cell count $\left(>100 \times 10^{9} / 1\right)$, and $86 \%(\mathrm{n}=32)$ had advanced-stage Burkitt's lymphoma or lymphoblastic lymphomas. In addition, $97 \%$ of patients

Table 1 Baseline characteristics of critically ill children with haematological malignancies

\begin{tabular}{|c|c|c|c|c|}
\hline & $\begin{array}{l}\text { All patients } \\
(\mathrm{n}=37)\end{array}$ & $\begin{array}{l}\text { Standard-dose group } \\
(>0.1 \mathrm{mg} / \mathrm{kg} / \text { day }) \\
(\mathrm{n}=22)\end{array}$ & $\begin{array}{l}\text { Low-dose group } \\
(\leq 0.1 \mathrm{mg} / \mathrm{kg} / \text { day }) \\
(\mathrm{n}=15)\end{array}$ & $P$ value \\
\hline Age & $6.4 \pm 3.4$ & $5.1 \pm 3.1$ & $8.3 \pm 2.8$ & $0.002^{*}$ \\
\hline \multicolumn{5}{|l|}{ Sex } \\
\hline Male & 28 & 16 & 12 & \multirow[t]{2}{*}{0.908} \\
\hline Female & 9 & 6 & 3 & \\
\hline Weight & $22.3 \pm 10.9$ & $17.8 \pm 8.3$ & $28.9 \pm 11.0$ & $0.001 *$ \\
\hline Disease course & $35 \pm 26$ & $35 \pm 27$ & $35 \pm 25$ & 0.999 \\
\hline \multicolumn{5}{|l|}{ Rasburicase exposure } \\
\hline Total dose $(\mathrm{mg} / \mathrm{kg})$ & $0.58 \pm 0.42$ & $0.76 \pm 0.41$ & $0.33 \pm 0.29$ & $0.001^{*}$ \\
\hline Daily dose (mg/kg/day) & $0.13 \pm 0.06$ & $0.16 \pm 0.04$ & $0.08 \pm 0.02$ & $<0.001^{*}$ \\
\hline Frequency (times) & $4.58 \pm 2.97$ & $4.89 \pm 2.98$ & $4.13 \pm 3.00$ & 0.456 \\
\hline \multicolumn{5}{|l|}{ Tumour histology } \\
\hline Burkitt’s lymphoma & 30 & 19 & 11 & \multirow[t]{4}{*}{0.405} \\
\hline Lymphoblastic lymphoma & 2 & 1 & 1 & \\
\hline Acute lymphoblastic Leukaemia & 4 & 1 & 3 & \\
\hline Acute myeloid Leukaemia & 1 & 1 & 0 & \\
\hline \multicolumn{5}{|l|}{ Laboratory values at admission } \\
\hline BUN (mmol/L) & $4.68 \pm 2.38$ & $4.89 \pm 2.74$ & $4.38 \pm 1.77$ & 0.529 \\
\hline $\mathrm{SCr}(\mu \mathrm{mol} / \mathrm{L})$ & $55 \pm 29$ & $55 \pm 33$ & $55 \pm 23$ & 0.976 \\
\hline $\mathrm{UA}(\mu \mathrm{mol} / \mathrm{L})$ & $677 \pm 288$ & $625 \pm 291$ & $754 \pm 274$ & 0.184 \\
\hline LDH (U/L) & $2179(1137,3282)$ & $1871(1056,2768)$ & $2805(1265,4586)$ & 0.234 \\
\hline
\end{tabular}

$B U N$ Blood urea nitrogen, $S C r$ serum creatinine, $U A$ uric acid, $L D H$ lactate dehydrogenase; $* P<0.05$ 
$(\mathrm{n}=36)$ had other risk factors for TLS at admission, such as renal involvement $(\mathrm{n}=3,8 \%)$, renal dysfunction $(\mathrm{n}=14,38 \%), \mathrm{LDH} \geq 2 \times$ upper limit of normal (ULN) $(\mathrm{n}=36,97 \%)$, hyperuricaemia $(\mathrm{n}=27,72.97 \%)$, hyperkalaemia $(\mathrm{n}=1,3 \%)$ and hyperphosphatemia $(\mathrm{n}=1$, $3 \%$ ). According to the clinical TLS risk assessment score proposed by Michael et $\mathrm{al}^{9}, 24 \%(\mathrm{n}=9)$ of patients were at very high risk of clinical TLS, while $56 \%(n=5)$ of them developed clinical TLS (Table 3). The mean risk assessment score was $9.5 \pm 5.1$. No significant difference in scores was seen between the different treatment groups $(9.0 \pm 4.5$ vs. $10.1 \pm 6.0, P=0.560)$.

\section{Rasburicase exposure}

All patients received at least one dose of rasburicase, 30 cases in the pre-chemotherapy period within D-1 to D1 because of hyperuricaemia and 7 cases within D3 to D6 due to TLS or AKI during chemotherapy. Details of the rasburicase exposures are also summarized in Table 1. The mean dose of rasburicase was $0.13 \pm 0.06 \mathrm{mg} / \mathrm{kg} /$ day. Twenty-two cases in the standard-dose group received dosages in the range of $0.11 \mathrm{mg} / \mathrm{kg} / \mathrm{day}$ to $0.20 \mathrm{mg} / \mathrm{kg} /$ day, while 15 cases in the low-dose group varied from $0.04 \mathrm{mg} / \mathrm{kg} / \mathrm{day}$ to $0.10 \mathrm{mg} / \mathrm{kg} / \mathrm{day}$. Eighty-one percent (30/37) of patients needed at least one more dose of rasburicase. One patient in the low-dose group who had no history of allergy or drug hypersensitivity developed anaphylactic shock after the first dose of rasburicase. No other side effects were observed during rasburicase treatment. In those patients with hyperuricaemia, the uric acid (UA) levels of 3 patients in the low-dose group decreased to normal in 36-72 h, while that of the others (27/30) returned to the normal range within $12 \mathrm{~h}$. Changes in UA levels after the first dose of rasburicase are shown in Fig. 1.

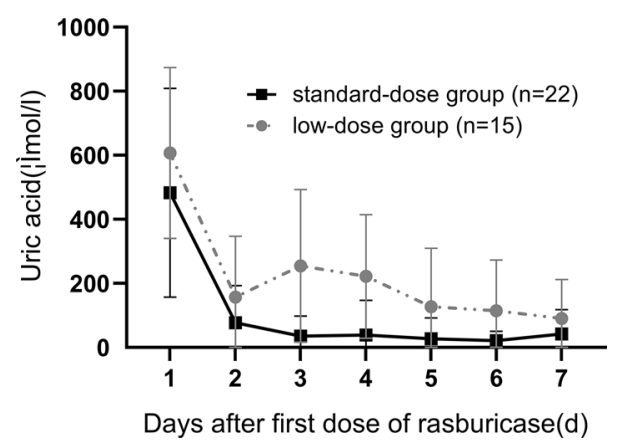

Fig. 1 Rapid decrease in uric acid level after first dose of rasburicase in each groups. The line graph shows mean standard deviations of the uric acid decrease level

\section{Clinical outcome}

All 37 patients received pre-phase chemotherapy, as established by the South China Children's Cancer Group (SCCCG), after histologically confirmed haematological malignancy to reduce tumour load $[7,14]$. The induction treatment was adjusted in 5 patients. Three patients needed to postpone formal chemotherapy because of multiple organ dysfunction. Two patients with Burkitt's lymphoma had delayed highdose methotrexate treatment as a subsequent regimen due to massive ascites. The median follow-up time was 13 months (2-29 months). Clinical outcomes are listed in Table 2.

Serious complications were frequent in critically ill children with haematological malignancies $(n=31,84 \%)$, and tumour lysis syndrome was the most common complication $(\mathrm{n}=27,73 \%)$. The median occurrence time of TLS was D3 (D3-D5). Hyperuricaemia (25/27) and hyperphosphatemia (17/27) were prevalent symptoms of TLS, which may lead to the high incidence of AKI. Forty-three percent of patients developed clinical TLS. The incidence of clinical TLS, stratified according to the clinical TLS risk assessment score, is shown in Table 3. There was no significant difference between the standard-dose group and the lowdose group. Fifty-nine percent of patients had AKI, 41\% (9/22) of whom required renal replacement therapy (RRT), 5 in the standard-dose group and 4 in another group, with a median RRT time of 3 days. The recorded causes for haemodialysis were respiratory failure $(\mathrm{n}=5,2$ cases combined with congestive heart failure) and severe acidosis with hyperlactatemia $(n=4)$. None of the surviving AKI patients required long-term haemodialysis or progressed to chronic kidney disease. The median renal function recovery time was 4.0 days (2.5-6.0 days), with no significant difference between groups $(P=0.802)$. Other serious complications, including respiratory failure $(\mathrm{n}=9,24 \%)$, disseminated intravascular coagulation $(\mathrm{n}=6,16 \%)$ and heart failure $(\mathrm{n}=4,11 \%)$, occurred in $27 \%$ of patients.

The overall 7-day and 28-day survival rates after ICU admission were $86 \%$ and $84 \%$, respectively. Six patients died because of cerebral herniation $(n=3,8 \%)$, cardiac arrest caused by clinical TLS $(\mathrm{n}=2,5 \%)$, and septic shock $(\mathrm{n}=1$, $3 \%$ ). The other 31 patients are currently alive. The average length of stay in the ICU was $9.92 \pm 5.13$ days. Neither length nor cost of stay in the ICU was significantly different between the two groups. The overall survival curves of the 37 patients are shown in Fig. 2.

\section{Discussion}

A number of studies have confirmed that rasburicase is safe and effective in the treatment of TLS in children. Although rasburicase is recommended for patients categorized as 
Table 2 Serious compilations and prognosis of critically ill children with haematological malignancies
Table 3 Risk assessment and the incidence of clinical tumour lysis syndrome in critically ill children with haematological malignancies

\begin{tabular}{lllll}
\hline & $\begin{array}{l}\text { All patients } \\
(\mathrm{n}=37)\end{array}$ & $\begin{array}{l}\text { Standard-dose group } \\
(>0.1 \mathrm{mg} / \mathrm{kg} / \text { day }) \\
(\mathrm{n}=22)\end{array}$ & $\begin{array}{l}\text { Low-dose group } \\
(\leq 0.1 \mathrm{mg} / \mathrm{kg} / \text { day }) \\
(\mathrm{n}=15)\end{array}$ & P value \\
\hline $\begin{array}{l}\text { Incidence of serious com- } \\
\text { plications }\end{array}$ & $84 \%(31)$ & $77 \%(17)$ & $93 \%(14)$ & 0.397 \\
TLS & $73 \%(27)$ & $73 \%(16)$ & $73 \%(11)$ & 1.000 \\
Laboratory TLS & $30 \%(11)$ & $23 \%(5)$ & $40 \%(6)$ & 0.417 \\
Clinical TLS & $43 \%(16)$ & $50 \%(11)$ & $33 \%(5)$ & 0.500 \\
AKI & $59 \%(22)$ & $64 \%(14)$ & $53 \%(8)$ & 0.775 \\
Stage 1 & $11 \%(4)$ & $9 \%(2)$ & $13 \%(2)$ & \\
Stage 2 & $16 \%(6)$ & $14 \%(5)$ & $7 \%(1)$ & \\
Stage 3 & $32 \%(12)$ & $32 \%(7)$ & $33 \%(5)$ & 1.000 \\
Requirement of RRT & $24 \%(9)$ & $23 \%(5)$ & $27 \%(4)$ & 0.908 \\
Respiratory failure & $24 \%(9)$ & $27 \%(6)$ & $7 \%(1)$ & 0.896 \\
Heart failure & $11 \%(4)$ & $14 \%(3)$ & $20 \%(3)$ & 0.670 \\
DIC & $16 \%(6)$ & $14 \%(3)$ & $20 \%(3)$ & 0.643 \\
Others & $14 \%(5)$ & $9 \%(2)$ & & \\
Prognosis & & & $20 \%$ & 0.643 \\
7-day mortality & $14 \%$ & $9 \%$ & $8.27 \pm 4.93$ & 0.951 \\
28-day mortality & $16 \%$ & $14 \%$ & $9971 \pm 6556$ & 0.482 \\
LOS in ICU (d) & $8.95 \pm 4.77$ & $9.41 \pm 4.71$ & & 0.978 \\
Costs in ICU stay (\$) & $10003 \pm 4949$ & $10024 \pm 3651$ & & \\
\hline
\end{tabular}

$T L S$ tumour lysis syndrome, $A K I$ acute kidney injury, RRT renal replacement therapy, DIC Disseminated intravascular coagulation, $L O S$ length of stay, $I C U$ intensive care unit

\begin{tabular}{|c|c|c|c|c|c|c|c|}
\hline \multirow[t]{2}{*}{ Risk category } & \multirow[t]{2}{*}{ Score } & \multicolumn{3}{|c|}{ Risk assessment of Clinical TLS } & \multicolumn{3}{|c|}{ Incidence of Clinical TLS } \\
\hline & & All & S-D group & L-D group & All & S-D group & L-D group \\
\hline Low risk & $\leq 5$ & 12 & 7 & 5 & 2 & 1 & 1 \\
\hline Intermediate risk & $6-9$ & 9 & 6 & 3 & 4 & 3 & 1 \\
\hline High risk & $10-13$ & 7 & 4 & 3 & 4 & 3 & 1 \\
\hline Very high risk & $\geq 14$ & 9 & 5 & 4 & 6 & 4 & 2 \\
\hline
\end{tabular}

$T L S$ tumour lysis syndrome, $S$-D group: standard-dose group (rasburicase $>0.1 \mathrm{mg} / \mathrm{kg} / \mathrm{day}$ ), $L$ - $D$ group low-dose group (rasburicase $\leq 0.1 \mathrm{mg} / \mathrm{kg} /$ day)

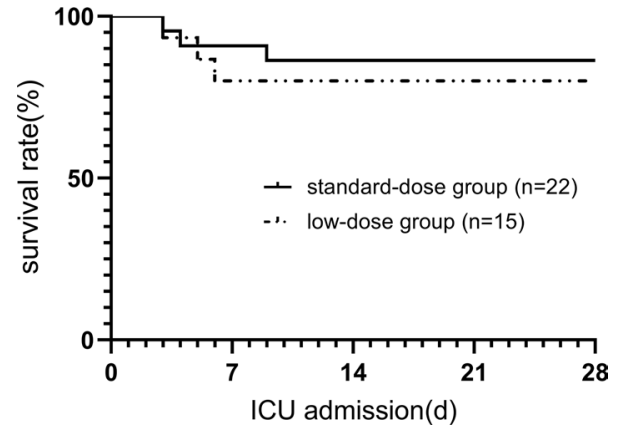

Fig. 2 The Kaplan-Meier survival analysis for days from ICU admission to death $(\mathrm{P}=0.610)$ being at the highest risk of developing TLS, at present, there is still great variability in the use of rasburicase in different countries and regions. A cross-sectional study showed that the utilization rate of rasburicase exposure from 48 children's hospitals throughout the United States was from 7\% to $71 \%$ [15] and that it is estimated to be even lower in developing countries [16], far from the application recommended by the guidelines [15]. In our institution, 57\% of children with haematological malignancies used rasburicase during their ICU stay. Rasburicase was used only in high-risk patients. A more stringent standard was applied in rasburicase therapy that in $97 \%$ of patients with more than one risk factor for TLS. Most patients $(\mathrm{n}=27,73 \%)$ even had $\geq 3$ risk factors.

The recommended dose of rasburicase is $0.2 \mathrm{mg} / \mathrm{kg}$ for 5-7 days, signifying a higher cost than the cost of the standard care for TLS [5], which may be one of the reasons 
for its limited application. Recent studies about reducing the frequency of rasburicase administration indicated that single-dose rasburicase also has clinical efficacy and can save on drug costs in the treatment of TLS $[9,17,18]$, but $21-33 \%$ of patients who are at high risk of TLS require at least two doses of rasburicase due to rebound of uric acid $[10,19]$. Our study shows that the proportion of patients requiring a second dose of rasburicase appears to be higher in critically ill children. The requirement of repeated doses of rasburicase may result from the continuous disintegration of tumour cells and the production of uric acid in the early stage of chemotherapy, which lasts far longer than the duration of rasburicase action. Previous studies have shown that plasma uric acid drops to an undetectable level in $84 \%$ of patients after the first recommended dose of rasburicase [17]. Because rasburicase is expensive and a selffinanced drug in our country, the expenses are beyond the financial reach of many households. Thus, we compared different dosing strategies of rasburicase to explore the optimal dosage for maximum pharmacoeconomic benefits. However, the main limitation in previous studies is that they only focused on the decline of uric acid rather than on the prognosis of the children. Information about the incidence of serious adverse events and the outcomes of such patients is indistinct, especially in critically ill children. A study on patients with malignancies who underwent chemotherapy in the ICU showed that the in-ICU incidence of TLS was 36\%, much higher than that in the general ward $(0.02 \%)$. AKI and the requirement of RRT are also more common in those patients, suggesting that the prevention and treatment of TLS are more challenging in ICU patients [20]. While comparing two groups with different initial doses of rasburicase, whether greater or less than $0.1 \mathrm{mg} / \mathrm{kg}$, we found that both the incidence of severe complications and the survival rate of patients were comparable between the two groups, which suggested that a smaller initial dose of rasburicase could be considered for children in the ICU.

The incidence of TLS and AKI in this study is high, which may be related to the more complicated condition of critically ill children. In addition, the prevalence of hyperuricaemia and hyperphosphatemia may also partly explain the high incidence of AKI [21]. Nevertheless, our study shows lower mortality in critically ill patients with TLS, which may be due to the wide application of rasburicase treatment [20]. There was no difference in the incidences of TLS and AKI between different dose groups, suggesting that a low dose of rasburicase is safe and effective in critically ill children with a high tumour burden. However, further prospective studies with larger sample sizes are required to explore the efficacy of low-dose rasburicase therapy in preventing TLS and protecting renal function in critically ill children with large tumour burdens.
Prolonged ICU stays not only increase psychological stress and economic burden on the patients' families but also make follow-up treatment difficult. A few studies have demonstrated that reducing the rasburicase dosage may reduce the cost of hyperuricaemia treatment [10,22], but there is a lack of research concern about the total financial burden of ICU costs. In our study, no significant difference in in-ICU expenses was observed between the two groups, indicating that the reduction of the rasburicase dose not increase inICU expenses. This is consistent with the result in our study that low-dose rasburicase does not increase life-threatening situations. Children in the low-dose group tended to have heavier body weights; thus, the dose and cost of other drugs would increase correspondingly, which may contribute to the negative result. Although patients in the low-dose group showed a reduction trend in the in-ICU cost, more studies are needed to confirm the role of low-dose rasburicase in reducing the economic burden.

\section{Conclusion}

Tumour lysis syndrome is a potentially life-threatening complication and one of the most common causes of shortterm death in children with a large tumour burden in intensive care units. Though limited, the findings from our study indicate that using less than the recommended dose of rasburicase may be an acceptable choice for critically ill children with haematological malignancies, especially in resource-limited areas.

Acknowledgements We thank all the members of the Pediatric Critical Tumor Treatment Team, including the Pediatric Oncology Department of the Sun Yat-sen University Cancer Center and the Pediatric Intensive Care Unit and Pediatric Surgery Department of the First Affiliated Hospital of Sun Yat-sen University, for assistance.

Funding This work was supported by the Medical Research Foundation of Guangdong Province [Grant Number A2019375]. The funding source did not have any role in the design, execution, analysis, interpretation, or writing of the report or in the decision to submit this report for publication. The corresponding author had full access to all the study data and had final responsibility for the decision to submit.

Data and/or Code availability The datasets generated during and/or analysed during the present study are available from the corresponding author on reasonable request.

Conflicts of interest The authors have declared no conflicts of interest.

Ethics approval This retrospective chart review study involving human participants was in accordance with the ethical standards of the institutional and national research committee and with the 1964 Helsinki Declaration and its later amendments or comparable ethical standards. The Medical Ethics Committee of the First Affiliated Hospital of Sun Yat-sen University approved this study [No.2020-092]. 
Open Access This article is licensed under a Creative Commons Attribution 4.0 International License, which permits use, sharing, adaptation, distribution and reproduction in any medium or format, as long as you give appropriate credit to the original author(s) and the source, provide a link to the Creative Commons licence, and indicate if changes were made. The images or other third party material in this article are included in the article's Creative Commons licence, unless indicated otherwise in a credit line to the material. If material is not included in the article's Creative Commons licence and your intended use is not permitted by statutory regulation or exceeds the permitted use, you will need to obtain permission directly from the copyright holder. To view a copy of this licence, visit http://creativecommons.org/licenses/by/4.0/.

\section{References}

1. Cairo MS, Bishop M. Tumour lysis syndrome: new therapeutic strategies and classification. Br J Haematol. 2004;127(1):3-11. https://doi.org/10.1111/j.1365-2141.2004.05094.x.

2. Rahmani B, Patel S, Seyam O, Gandhi J, Reid I, Smith N, et al. Current understanding of tumor lysis syndrome. Hematol Oncol. 2019;37(5):537-47. https://doi.org/10.1002/hon.2668.

3. Cheuk DK, Chiang AK, Chan GC, Ha SY. Urate oxidase for the prevention and treatment of tumour lysis syndrome in children with cancer. Cochrane Database Syst Rev. 2017;3(3):CD006945. https://doi.org/10.1002/14651858.cd006945.pub4.

4. Cairo MS, Coiffier B, Reiter A, Younes A. Recommendations for the evaluation of risk and prophylaxis of tumour lysis syndrome (TLS) in adults and children with malignant diseases: an expert TLS panel consensus. Br J Haematol. 2010;149(4):578-86. https ://doi.org/10.1111/j.1365-2141.2010.08143.x.

5. Hu S, Han Y, Zhang W, Zhang T, Yao X, Liu L. Cost-effectiveness analysis of rasburicase over standard of care for the prevention and treatment of tumor lysis syndrome in children with hematologic malignancies in China. J Med Econ. 2019;22(8):742-50. https:// doi.org/10.1080/13696998.2019.1603155.

6. Savva DA, Herrera N, Rohatgi R. Comparison of fixed versus traditional weight-based dosing of rasburicase in a pediatric population. Pediatr Blood Cancer. 2018;65(10):e27236. https://doi. org/10.1002/pbc.27236.

7. Sun XF, Zhen ZJ, Xia Y, Lin SX, Zhu J, Wang J et al. [Outcome of children and adolescents with Burkitt lymphoma and diffuse large B cell lymphoma treated with a modified NHL-BFM-90 protocol]. Zhonghua xue ye xue za $\mathrm{zhi}=$ Zhonghua xueyexue zazhi. 2013;34(12):1032-7. https://doi.org/10.3760/cma.j.i ssn.0253-2727.2013.12.008.

8. Cairo MS, Sposto R, Perkins SL, Meadows AT, Hoover-Regan ML, Anderson JR, et al. Burkitt's and Burkitt-like lymphoma in children and adolescents: a review of the Children's Cancer Group experience. Br J Haematol. 2003;120(4):660-70. https://doi.org/ 10.1046/j.1365-2141.2003.04134.x.

9. Philips A, Radhakrishnan V, Ganesan P, Ganesan TS, Ramamurthy J, Dhanushkodi M, et al. Efficacy of Single Dose Rasburicase $(1.5 \mathrm{mg})$ for Prophylaxis and Management of Laboratory Tumor Lysis Syndrome. Indian J Hematol Blood Transf. 2018;34(4):61822. https://doi.org/10.1007/s12288-018-0938-9.

10. Syrimi E, Gunasekera S, Norton A, Velangi M, Motwani J, Hiwarkar P. Single dose Rasburicase is a clinically effective pharmacoeconomic approach for preventing tumour lysis syndrome in children with high tumour burden. Br J Haematol. 2018;181(5):696-8. https://doi.org/10.1111/bjh.14689.

11. Jones GL, Will A, Jackson GH, Webb NJ, Rule S. Guidelines for the management of tumour lysis syndrome in adults and children with haematological malignancies on behalf of the British Committee for Standards in Haematology. Br J Haematol. 2015;169(5):661-71. https://doi.org/10.1111/bjh.13403.

12. Darmon M, Vincent F, Camous L, Canet E, Bonmati C, Braun $\mathrm{T}$, et al. Tumour lysis syndrome and acute kidney injury in highrisk haematology patients in the rasburicase era. A prospective multicentre study from the Groupe de Recherche en Réanimation Respiratoire et Onco-Hématologique. Br J Haematol. 2013;162(4):489-97. https://doi.org/10.1111/bjh.12415.

13. Kellum JA, Lameire N. Diagnosis, evaluation, and management of acute kidney injury: a KDIGO summary (Part 1). Critical Care (London, England). 2013;17(1):204. https://doi.org/10.1186/ cc11454.

14. Fang JP, Luo XQ, Tu LM, Lai DB, Sun XF, Chen JL, et al. A multiple-center clinical study on the therapy for childhood acute lymphoblastic leukemia by using GZ-2002 ALL protocol. J China Pediat Blood Cancer. 2011;16(2):18-23. https://doi.org/10.3969/j. issn.1673-5323.2011.02.005.

15. Citrin R, Getz KD, Li Y, Huang YS, Horowitz JP, Seif AE, et al. The epidemiology of rasburicase use in paediatric patients with acute lymphoblastic leukaemia and non-Hodgkin lymphoma. Br J Haematol. 2019;184(4):684-8. https://doi.org/10.1111/bjh.15159

16. Gopakumar KG, Seetharam S, Km JK, Nair M, Rajeswari B, Cs G, et al. Risk-based management strategy and outcomes of tumor lysis syndrome in children with leukemia/lymphoma: analysis from a resource-limited setting. Pediatr Blood Cancer. 2018;65(12):e27401. https://doi.org/10.1002/pbc. 27401.

17. Vadhan-Raj S, Fayad LE, Fanale MA, Pro B, Rodriguez A, Hagemeister FB, et al. A randomized trial of a single-dose rasburicase versus five-daily doses in patients at risk for tumor lysis syndrome. Ann Oncol. 2012;23(6):1640-5. https://doi.org/10.1093/annonc/ mdr490.

18. McDonnell AM, Lenz KL, Frei-Lahr DA, Hayslip J, Hall PD. Single-dose rasburicase $6 \mathrm{mg}$ in the management of tumor lysis syndrome in adults. Pharmacotherapy. 2006;26(6):806-12. https ://doi.org/10.1592/phco.26.6.806.

19. Gopakumar KG, Thankamony P, Seetharam S, P K. Treatment of tumor lysis syndrome in children with leukemia/lymphoma in resource-limited settings: Efficacy of a fixed low-dose rasburicase. Pediatric Hematol Oncol. 2017;34(4):206-11. https://doi. org/10.1080/08880018.2017.1348415.

20. Wohlfarth P, Staudinger T, Sperr WR, Bojic A, Robak O, Hermann A, et al. Prognostic factors, long-term survival, and outcome of cancer patients receiving chemotherapy in the intensive care unit. Ann Hematol. 2014;93(10):1629-36. https://doi. org/10.1007/s00277-014-2141-x.

21. van den Berg H, Reintsema AM. Renal tubular damage in rasburicase: risks of alkalinisation. Ann Oncol. 2004;15(1):175-6. https ://doi.org/10.1093/annonc/mdh029.

22. Feng X, Dong K, Pham D, Pence S, Inciardi J, Bhutada NS Efficacy and cost of single-dose rasburicase in prevention and treatment of adult tumour lysis syndrome: a meta-analysis. J Clin Pharm Ther. 2013;38(4):301-8. https://doi.org/10.1111/ jept.12061.

Publisher's Note Springer Nature remains neutral with regard to jurisdictional claims in published maps and institutional affiliations. 